\title{
Gastroenteropancreatic neuroendocrine tumors. Where are we now?
}

\author{
Daniel Castellano
}

Published online: 2 March 2011

(C) Springer Science+Business Media, LLC 2011

Gastroenteropancreatic neuroendocrine tumors (GEPNETs) are tumors derived from neuroendocrine cells that can occur anywhere along the gastrointestinal tract. Due to their particular features, GEP-NETs are among the most complex tumors to diagnose and treat. For instance, symptoms vary according to the type of GEP-NET, and therefore they are easily mistaken for other conditions.

Although the general idea is that GEP-NETs are rare tumors, the most recent data from the US Surveillance Epidemiology and End Results show an impressive increase of more than $400 \%$ in the incidence of this disease over a period of 29 years, rising from 1.09 per 100,000 population in 1973 to 5.25 per 100,000 population in 2004 [1]. Only in the last year, their incidence increase from 3.00 to 5.25 per 100,000 . In fact, GEP-NETs are more prevalent than many other tumors of the gastrointestinal tract, including stomach and pancreatic carcinomas combined.

Survival of patients with GEP-NETs depends greatly on stage and histology. For example, patients with well and moderately well-differentiated distant metastases have a 5-year survival probability of $35 \%$; conversely, in patients with poorly differentiated distant metastases, the 5-year survival probability drops to only $4 \%$ [1]. The mere observation of these figures calls our attention to the importance of an early diagnosis, and tumor classification and staging of patients in whom a GEPNET is suspected, as well as to the need for ongoing modifications in the therapeutic approach.

D. Castellano $(\bowtie)$

Board Member of GETNE,

Departamento de Oncología Médica,

Hospital Universitario 12 de Octubre,

Avda. de Córdoba, s/n,

28041 Madrid, Spain

e-mail: cdanicas@hotmail.com
Interestingly, in parallel to changes in the epidemiology of GEP-NET, there have been substantial advances in treatment, such as the use of peptide receptor radiotherapy, and more recently, antiangiogenic agents (bevacizumab, everolimus, sunitinib), which have echoed on the prognosis of many patients with this disease. As shown by the results of the RADIANT trials, with the advent of targeted therapies, we may be on the verge of a paradigm shift in the treatment of advanced GEP-NETs.

The Spanish Group of Neuroendocrine Tumors (GETNE), aware of the interest this disease has raised due to its increasing incidence and significant advances in treatment, considered it would be of relevance to publish the contents from the " $V$ GETNE Symposium on GEPNETS" held in September 2009 in the city of Madrid (Spain). In this Special Issue, the updated contents of this symposium are reported. The purpose is to provide a general overview of the current state of knowledge of GEPNETs regarding diagnosis and innovations in therapy.

\section{Reference}

1. Yao, J. C., Hassan, M., Phan, A., Dagohoy, C., Leary, C., Mares, J. E., et al. (2008). One hundred years after "carcinoid": epidemiology of and prognostic factors for neuroendocrine tumors in 35,825 cases in the United States. Journal of Clinical Oncology, 26(18), 3063-72. 